Nota científica

\title{
Primer registro de la angiosperma marina Halophila ovalis en las Antillas Mayores
}

\author{
First record for seagrass Halophila ovalis in the Greater Antilles \\ Joán I. Hernández-Albernas ${ }^{\text {a, } *}$ y Celia C. Borges-Casas ${ }^{b}$ \\ ${ }^{a}$ Refugio de fauna Cayo Santa María, Delegación Territorial Gaviota Centro, Carretera a Remedios, km. 1, 52610, Caibarién, Villa Clara, Cuba \\ ${ }^{\mathrm{b}}$ Facultad de Ciencias Agropecuarias, Universidad Central de Las Villas, Carretera a Camajuaní, km. 5.5, 50100, Santa Clara, Villa Clara, Cuba
}

Recibido el 20 de enero de 2017; aceptado el 17 de mayo de 2017

Disponible en Internet el 6 de diciembre de 2017

\begin{abstract}
Resumen
Se registra por primera vez la fanerógama Halophila ovalis (R. Brown) J. D. Hooker, en aguas cubanas. El análisis de caracteres morfológicos y ecológicos valida este registro, ampliando el área de distribución de esta especie en el Atlántico.

(C) 2017 Universidad Nacional Autónoma de México, Instituto de Biología. Este es un artículo Open Access bajo la licencia CC BY-NC-ND

(http://creativecommons.org/licenses/by-nc-nd/4.0/).
\end{abstract}

Palabras clave: Halophila; Pastos marinos; Cuba

\begin{abstract}
The seagrass Halophila ovalis (R. Brown) J. D. Hooker, is reported for the first time from Cuban waters. Morphological and ecological features are according to published data about the species, broadening its distribution range along the Atlantic basin.

(C) 2017 Universidad Nacional Autónoma de México, Instituto de Biología. This is an open access article under the CC BY-NC-ND license

(http://creativecommons.org/licenses/by-nc-nd/4.0/).
\end{abstract}

Keywords: Halophila; Seagrass; Cuba

El género Halophila en la región atlántica está representado por 6 especies de plantas marinas con una distribución biogeográfica variable. H. decipiens posee distribución tropical mundial; $H$. baillonii tiene una distribución discontinua para el Atlántico tropical occidental, aunque también ha sido encontrada en las costas del Pacífico de Panamá, Costa Rica y Nicaragua (Short, Fernández, Vernon y Gaeckle, 2006; Tussenbroek, Barba-Santos, Ricardo-Wong, van Dijk y Waycott, 2010); H. engelmannii se reporta con discontinuidad para el Atlántico tropical occidental; H. johnsonii, con distribución muy restringida, es considerada una especie endémica para la costa oriental de la Florida.

\footnotetext{
* Autor para correspondencia.

Correo electrónico: esp1.medio @ cen.gaviota.cu (J.I. Hernández-Albernas).

La revisión por pares es responsabilidad de la Universidad Nacional Autónoma de México.
}

H. stipulacea y H. ovalis son las más recientes adiciones del género al inventario florístico en el Atlántico tropical, a partir de los años 2002 y 2010, respectivamente. Ambas especies, restringidas inicialmente al sublitoral de las Antillas Menores, han sido consideradas especies exóticas introducidas por acciones antrópicas como dragados o tráfico marítimo (Ruiz y Ballantine, 2004; Short, Moore y Peyton, 2010).

Halophila ovalis (R. Brown) J. D. Hooker es la especie de angiosperma marina más común en la región tropical y subtropical del Índico y las costas orientales del Pacífico. Ha sido catalogada como un complejo, bajo cuya denominación pudieran agruparse otras descritas, a partir de análisis de secuencias genómicas en el ADN ribosomal (Uchimura, Faye, Shimada, Inoue y Nakamura, 2008).

Short et al. (2010) la registraron para la cuenca del Atlántico, en la isla de Antigua, de las Antillas Menores, planteando que esta especie pudiese tener la capacidad natural para 


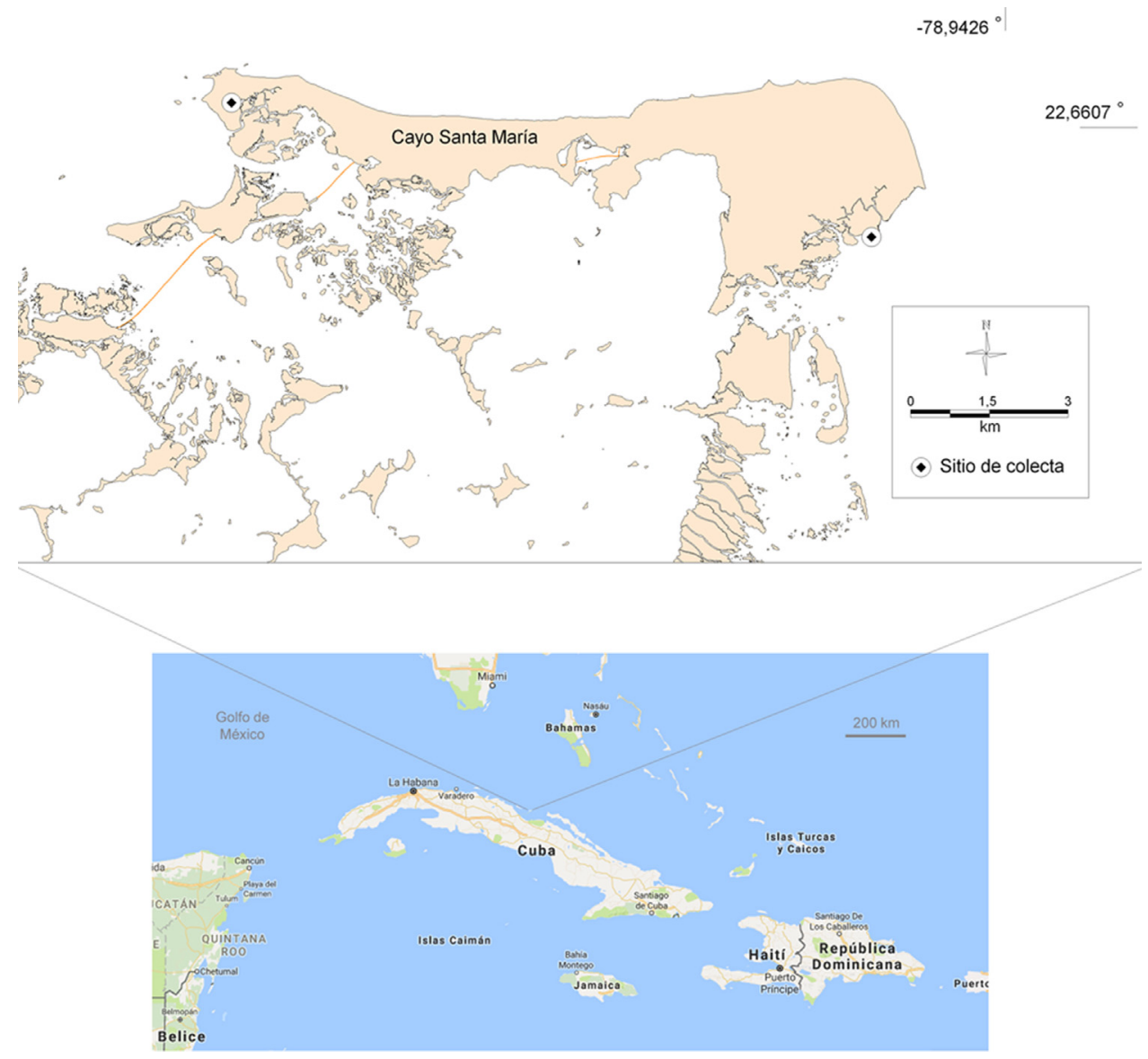

Figura 1. Ubicación del registro de Halophila ovalis en las Antillas Mayores.

dispersarse a grandes distancias, al considerar su bajo porcentaje de divergencia genómica. En esta nota se confirma su presencia por primera vez en aguas marinas cubanas, ampliando su rango de distribución en el Atlántico, a más de $1,800 \mathrm{~km}$ al noroeste del último reporte.

Los especímenes se detectaron el 21 de diciembre de 2016 , en 2 sitios del sublitoral somero: $22^{\circ} 39^{\prime} 47^{\prime \prime} \mathrm{N}, 79^{\circ} 05^{\prime} 12^{\prime \prime} \mathrm{O}$ (canal interior en bosque de Rhizophora mangle); $22^{\circ} 38^{\prime} 29^{\prime \prime}$ $\mathrm{N}, 78^{\circ} 57^{\prime} 57^{\prime \prime} \mathrm{O}$ (entre individuos de Rhizophora mangle); en Cayo Santa María, centronorte de Cuba (fig. 1). Se recolectaron manualmente 30 fragmentos independientes de varias plantas detectadas sobre fondo arenofangoso, en profundidades inferiores a los $0.4 \mathrm{~m}$, y se trasladaron en agua de mar para su identificación a posteriori en el laboratorio.

Se evaluaron varios caracteres morfológicos con valor definitorio para precisar el taxón (borde y forma de la hoja, longitud del peciolo y las escamas, nervadura), según criterios de den Hartog y Kuo (2006), Kuo y den Hartog (2001) y Phillips y Meñez (1988).

En los especímenes analizados, la morfología vegetativa muestra las hojas en pares, ovadas, con venas laterales llegando al nervio central, de peciolos usualmente largos que presentan pares de escamas pequeñas en la base, típico del género Halophila sección Halophila (Kuo y den Hartog, 2001) (fig. 2). No se encontraron estructuras reproductoras. Todo el material recolectado coincide con las descripciones morfológicas y eco- lógicas dadas para la especie $H$. ovalis, del género Halophila Du Petit-Thouars, perteneciente a la familia Hydrocharitaceae, agrupada actualmente en la clase Magnoliopsida (WoRMS, 2015). Las recolectas fueron depositadas en el Centro de Colecciones del Departamento de Biología de la Universidad Central de Las Villas con el identificador CCBUCLV-S4.

Esta angiosperma se presentó en lugares protegidos de las fuertes corrientes de marea y del oleaje, y se la encuentra compartiendo hábitat con $H$. engelmannii, citada para la región por Martínez-Daranas et al. (2013).

La especie más similar reportada en aguas cubanas es H. decipiens, presente en la zona (Martínez-Daranas et al., 2008; Perdomo y Suárez, 2004), fundamentalmente a profundidades mayores (2-30 m), pero que es excluida del análisis por presentar bordes serrados, a diferencia de H. ovalis (fig. 3). Tampoco se presta a confusión con $H$. stipulacea, pues esta posee bordes serrados y unas escamas grandes que cubren totalmente el peciolo a manera de vaina.

Las variaciones en los caracteres morfológicos cuantitativos de $H$. ovalis de nuestra nota se corresponden a lo publicado para esta especie por Short et al. (2010). Es relevante la similitud morfológica con $H$. johnsonii, difiriendo solamente en algunas medidas fenotípicas que pudieran ser resultado de la respuesta a algún factor ambiental del hábitat donde se está desarrollando (tabla 1). Short et al. (2010) no encontraron divergencias de nucleótidos para secuencias de ADN ribosomal 


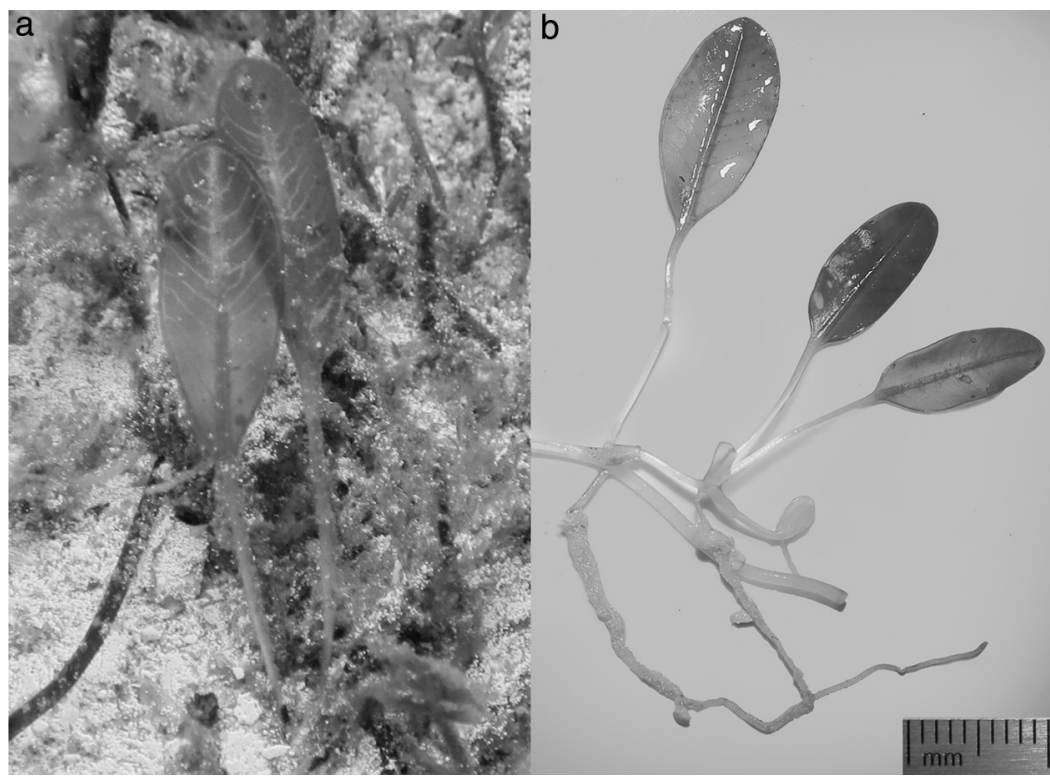

Figura 2. Estructuras vegetativas de Halophila ovalis: a) en su hábitat, b) en el laboratorio.
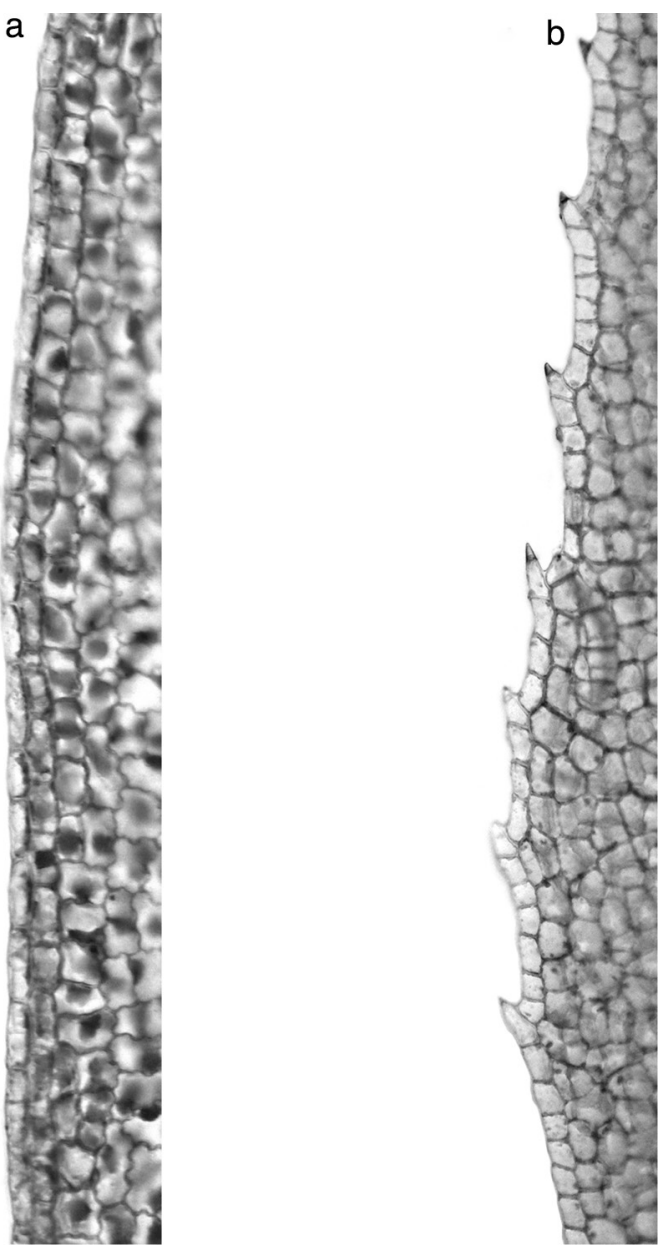

Tabla 1

Caracteres morfológicos evaluados en el material recolectado y Halophila johnsonii (Eiseman y McMillan, 1980).

\begin{tabular}{|c|c|c|c|}
\hline & \multicolumn{2}{|c|}{ Halophila ovalis } & \multirow{2}{*}{$\begin{array}{l}\text { Halophila johnsoni } \\
\text { Mínimo-máximo }\end{array}$} \\
\hline & Mínimo-máximo & Moda & \\
\hline Longitud de la hoja (mm) & $11-25$ & 16 & $5-25$ \\
\hline Ancho de la hoja (mm) & $5-10$ & 5 & $1-4$ \\
\hline Longitud del internodo (mm) & $9-33$ & 26 & $10-20$ \\
\hline Longitud de la escama (mm) & $3-6$ & 4 & $3-4$ \\
\hline Longitud del peciolo (mm) & $10-34$ & 19 & $10-20$ \\
\hline Pares de venas laterales & $8-14$ & 11 & $5-10$ \\
\hline Margen de la hoja & Entero (liso) & & Entero (liso) \\
\hline
\end{tabular}

(Uchimura et al., 2008; Waycott, Freshwater, York, Calladine y Kenworthy, 2002) entre ambas especies, lo cual evidencia la necesidad de revisión de la validez de las mismas como taxones diferentes.

Los autores expresan su agradecimiento a Manuel Faife y Luis Nivaldo por la colaboración en las recolectas, y a Beatriz Martínez y Arnaldo Toledo por la asistencia técnica.

\section{Referencias}

Den Hartog, C. y Kuo, J. (2006). Taxonomy and biogeography of seagrasses. En A. W. D. Larkum, R. J. Orth, y C. M. Duarte (Eds.), Seagrasses: biology, ecology and conservation (pp. 1-23). Dordrecht, The Netherlands: Springer.

Eiseman, N. J. y McMillan, C. (1980). A new species of seagrass, Halophila johnsonii, from the Atlantic coast of Florida. Aquatic Botany, 9, 15-19.

Kuo, J. y den Hartog, C. (2001). Seagrass taxonomy and identification key. En F. T. Short y R. G. Coles (Eds.), Global seagrass research methods (pp. 31-58). Amsterdam: Elsevier.

Martínez-Daranas, B., Cabrera, R., Perdomo, M. E., Esquivel, M., Hernández, M., Clero, L., et al. (2008). Inventario de la flora marina del archipiélago Sabana-Camagüey, Cuba. Botanica Complutensis, 32, 49-62.

Figura 3. Detalle del borde de la hoja: a) entero (material recolectado), b) serrado (Halophila decipiens).

Martínez-Daranas, B., Esquivel, M., Guimarais, M., Perdomo, M. E., Alfonso, Y., de la Guardia, E., et al. (2013). Distribución de Halophila engelmanni Ascherson (Hydrocharitaceae) en Cuba. Revista de Investigaciones Marinas, $33,21-27$. 
Perdomo, M. E. y Suárez, A. M. (2004). Fitobentos marino de Villa Clara, Cuba. Revista de Investigaciones Marinas, 25, 171-176.

Phillips, R. C. y Meñez, E. G. (1988). Seagrasses. Smithsonian Contributions to the Marine Sciences, 34, 1-104.

Ruiz, H. y Ballantine, D. L. (2004). Occurrence of the seagrass Halophila stipulacea in the tropical west Atlantic. Bulletin of Marine Science, 75, $131-135$.

Short, F. T., Fernández, E., Vernon, A. y Gaeckle, J. L. (2006). Ocurrence of Halophila baillonii meadows in Belize, Central America. Aquatic Botany, 85, 249-251.

Short, F. T., Moore, G. E. y Peyton, K. A. (2010). Halophila ovalis in the Tropical Atlantic Ocean. Aquatic Botany, 93, 141-146.

Uchimura, M., Faye, E. J., Shimada, S., Inoue, T. y Nakamura, Y. (2008). A reassessment of Halophila species (Hydrocharitaceae) diversity with special reference to Japanese representatives. Botanica Marina, 51, 258-268.

Van Tussenbroek, B. I., Barba-Santos, M. G., Ricardo-Wong, J. G., van Dijk, J. K. y Waycott, M. (2010). Guía de los pastos marinos tropicales del Atlántico oeste. México D. F.: UNAM.

Waycott, M., Freshwater, D. W., York, R. A., Calladine, A. y Kenworthy, W. J. (2002). Evolutionary trends in the seagrass genus Halophila (Thouars): insights from molecular phylogeny. Bulletin of Marine Science, 71, 1299-1308.

WoRMS (2015). Halophila ovalis (R. Brown) J. D. Hooker, 1858. En M. D. Guiry y G. M. Guiry. (2015). AlgaeBase. World-wide electronic publication, National University of Ireland, Galway (taxonomic information republished from AlgaeBase with permission of M. D. Guiry) [consultado 16 Ene 2017]. Disponible en http://marinespecies.org/aphia.php? $\mathrm{p}=$ taxdetailsyid=208930 